\title{
Le Kaizen et l'art de gérer la qualité médicale
}

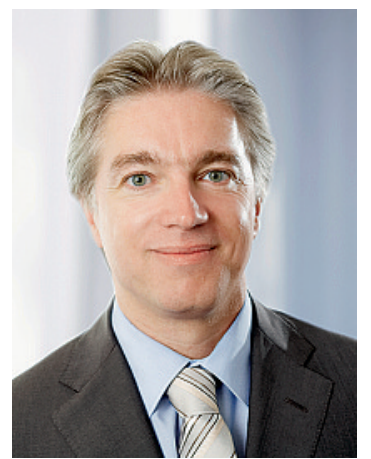

Daniel Herren
Le Kaizen est une notion de management d'origine japonaise. En traduction libre, cette notion signifie le changement (Kai) vers le mieux (Zen) et désigne ainsi une philosophie qui vise une amélioration progressive continue en opposition à un changement radical. C'est bien joli, me direz-vous, mais qu'est-ce que cela a à voir avec les prestations médicales? En y regardant de plus près, beaucoup de choses. La notion de qualité, telle qu'elle est perçue aujourd'hui par de nombreux acteurs, est en effet marquée par des idées statiques traditionnelles.

Certes, les mesures de la qualité servent à représenter une situation, mais elles n'offrent aucune solution. Par exemple, l'indication d'un taux de mortalité pour une intervention spécifique n'est utile que si, premièrement,

\section{La notion actuelle de qualité est marquée par des idées statiques traditionnelles}

cet indicateur est pertinent pour cette intervention (sur les plans quantitatif et qualitatif) et que, deuxièmement, il offre une possibilité de s'améliorer. C'est là que le Kaizen intervient. Dans le cadre d'un cycle continu, cette méthode cherche d'une part à conserver ce qui est bien, d'autre part à viser une amélioration continue dans les domaines problématiques. En ce qui concerne nos discussions courantes sur la mesure des prestations médicales, nous ferions bien de privilégier davantage le Kaizen à l'arithmétique des indicateurs.

Les mesures de la qualité doivent suivre une logique qui commence par saisir les activités qualité pour ensuite les valider et les évaluer. On ne peut évaluer de résultats qu'après avoir déterminé les activités utiles et judicieuses pour chaque groupe de prestataires et défini la méthode de mesure. Pour la FMH, il est essentiel de faire participer ces prestataires dès la phase d'évaluation. C'est sur cette base que des mesures pourront ensuite être définies en vue d'une amélioration durable de la qualité. Il peut s'agir

\section{En ce qui concerne la mesure des prestations médicales, nous ferions bien de privilégier davantage le Kaizen à I'arithmétique des indicateurs}

de récompenses, pas uniquement financières, motivant les prestataires à continuer de fournir des prestations de très grande qualité; il peut également s'agir de soutien aux prestataires qui n'accordent pas assez d'attention à la qualité et qui présentent un retard dans ce domaine. C'est pourquoi un système incitatif ne doit pas se limiter à prendre des mesures, mais doit prendre en compte l'intégralité du processus, par analogie avec l'idée du Kaizen. Tel est le fondement de notre idée du modèle «incentive for quality»: son potentiel réside, contrairement aux systèmes d'incitation financière, dans sa capacité à agir sans risque d'incitations inadéquates et de soins erronés.

Dr Daniel Herren MHA, membre du Comité central de la FMH, responsable du domaine $D D Q$

1 Pirsig RM. Zen und die Kunst ein Motorrad zu warten. Frankfurt: Fischer; 1974.

2 Imai M. Kaizen. Der Schlüssel zum Erfolg der Japaner im Wettbewerb. München: Langen-Müller; 1992.

3 Hutwelker R. hard und soft facts zur Prozessverbesserung. 2005. www.activepartner.de $\rightarrow$ Prozesse \& Ressourcen $\rightarrow$ KAIZEN/KVP $\rightarrow$ Materialien $\rightarrow$ Prozessverbesserungen mit KAIZEN/ KVP (Artikel) 